\title{
Cannabis y los sistemas exocannabinoide y endocannabinoide. Su uso y controversias
}

\author{
Rebeca Olivia Millán-Guerrero * y Sara Isais-Millán² \\ ${ }^{1}$ Universidad de Colima, Facultad de Medicina, Colima; ${ }^{2}$ Instituto de Seguridad y Servicios Sociales de los Trabajadores del Estado, Hospital \\ Regional "Ignacio Zaragoza", Servicio de Neurología, Ciudad de México. México
}

\begin{abstract}
Resumen
El cannabis (marihuana) es una de las sustancias psicoactivas más consumidas en el mundo. El término marihuana es de origen mexicano. Los cannabinoides primarios estudiados hasta la fecha incluyen el cannabidiol y el delta-9-tetrahidrocannabinol ( $\triangle 9-T H C)$, responsable de la mayoría de los efectos físicos y psicotrópicos del cannabis. Recientemente se descubrió el sistema endocannabinoide formado por receptores, ligandos y enzimas expresados ampliamente en el cerebro y su periferia, donde actúan para mantener el equilibrio en varios procesos homeostáticos. Los cannabinoides exógenos o fitocannabinoides de origen natural interactúan con el sistema endocannabinoide. La marihuana debe ser procesada en un laboratorio para extraer el tetrahidrocannabinol y dejar el cannabidiol, el producto que se puede comercializar. Algunos estudios otorgan al cannabidiol un gran potencial para el uso terapéutico como antiepiléptico, analgésico, ansiolítico, antipsicótico, antiinflamatorio y neuroprotector, sin embargo, son inconsistentes los hallazgos sobre la eficacia de los cannabinoides y la tolerabilidad-seguridad de los medicamentos con base en cannabis para cualquier padecimiento. Se requiere más evidencia científica para generar recomendaciones sobre el uso del cannabis medicinal.
\end{abstract}

PALABRAS CLAVE: Sustancias psicoactivas. Cannabinoides. Sistema endocannabinoide.

\begin{abstract}
Cannabis (marijuana) is one of the most consumed psychoactive substances in the world. The term marijuana is of Mexican origin. The primary cannabinoids that have been studied to date include cannabidiol and delta-9-tetrahydrocannabinol, which is responsible for most cannabis physical and psychotropic effects. Recently, the endocannabinoid system was discovered, which is made up of receptors, ligands and enzymes that are widely expressed in the brain and its periphery, where they act to maintain balance in several homeostatic processes. Exogenous cannabinoids or naturally-occurring phytocannabinoids interact with the endocannabinoid system. Marijuana must be processed in a laboratory to extract tetrahydrocannabinol and leave cannabidiol, which is the product that can be marketed. Some studies suggest cannabidiol has great potential for therapeutic use as an agent with antiepileptic, analgesic, anxiolytic, antipsychotic, anti-inflammatory and neuroprotective properties; however, the findings on cannabinoids efficacy and cannabis-based medications tolerability-safety for some conditions are inconsistent. More scientific evidence is required in order to generate recommendations on the use of medicinal cannabis.
\end{abstract}

KEY WORDS: Psychoactive substances. Cannabinoids. Endocannabinoid system.

Correspondencia:

*Rebeca Olivia Millán-Guerrero

E-mail: millanrebeca@hotmail.com
Fecha de recepción: 03-12-2018

Fecha de aceptación: 02-05-2019

DOI: 10.24875/GMM.19004881
Gac Med Mex. 2019;155:508-512

Disponible en PubMed www.gacetamedicademexico.com 


\section{Introducción}

El cannabis (marihuana) es una de las sustancias psicoactivas más consumidas en el mundo. El término marihuana es de origen mexicano. Por mucho tiempo, los botánicos creyeron que el cannabis y el cáñamo eran dos especies. Existen tres usos de la planta:

- En las zonas templadas, el cáñamo tiene una larga historia de su empleo en la fabricación de ropa, productos industriales, materiales de construcción, cuerdas, velas para navíos, pintura, barniz, solvente, combustible, papel, jabón, champú, cosméticos; incluso se consume como alimento. ${ }^{1}$

- Uso de la planta-droga por propiedades terapéuticas como analgésico, somnífero, antitusígeno, antiepiléptico, etcétera.

- Uso recreativo, hedónico y místico.

Las hojas y flores del cannabis contienen al menos 489 compuestos y 100 fitocannabinoides diferentes. ${ }^{2}$ Hay tres especies principales de cannabis: Cannabis sativa, Cannabis indica y Cannabis ruderalis. ${ }^{3}$ Los cannabinoides primarios estudiados hasta la fecha incluyen delta-9-tetrahidrocannabinol $(\Delta 9-\mathrm{THC}$, el más estudiado, responsable de la mayoría de los efectos físicos y psicotrópicos del cannabis), cannabidiol, cannabinol, cannabigerol y tetrahidrocannabivarin. ${ }^{4}$ Los cannabinoides más abundantes que la planta produce en diferentes proporciones son $\Delta$ 9-THC y cannabidiol. ${ }^{5}$ Los fitocannabinoides (cannabinoides de origen vegetal) han sido usados por milenios y de ellos se aislaron los ligandos cannabinoides exógenos tetrahidrocannabinol y cannabidiol. ${ }^{6}$

Recientemente se descubrió el sistema endocannabinoide, consiste en receptores, ligandos y enzimas expresados ampliamente en el cerebro y la periferia (Tabla 1), donde actúan para mantener el equilibrio en varios procesos homeostáticos. ${ }^{7,8}$

\section{Receptores}

Un receptor acoplado a proteína $G$ fue aislado de células de neuroblastoma murino, se denominó receptor de cannabinoides tipo 1 (CB1), ${ }^{9}$ el cual es el receptor acoplado a proteína $\mathrm{G}$ más abundante expresado en el cerebro de los mamíferos, con función neuromoduladora; se encuentra en diversos grados en todas las estructuras cerebrales y en la periferia de casi todos los órganos del cuerpo de los mamíferos. ${ }^{8}$ El CB1 se expresa a nivel presináptico (en terminales de neuronas glutamatérgicas y gamma-aminobutíricas-ergéticas) y postsináptico, asociado con astrocitos, para regular la liberación de neurotransmisores. ${ }^{10}$

A partir de células de leucemia humana se aisló el receptor de cannabinoides tipo 2 (CB2), que se encuentra en abundancia en las células inmunes, en microglía y astrocitos activados.

El receptor transitorio potencial vanilloid-1 (TRPV1), conocido como receptor de la capsaicina (el ingrediente activo de los chiles), está unido a un número de ligandos más allá de la capsaicina, incluidos los ligandos endocannabinoides, y puede activarse mediante estímulos físicos y mecánicos como pH bajo, altas temperaturas o cambios en la concentración osmótica.

Por último, el receptor GPR55, acoplado a la proteína $\mathrm{G} 55$, tiene un ligando endógeno, el lisofosfatidilinositol; su papel no está claro, se expresa en áreas corticales, cuerpo estriado, hipocampo y cerebelo y produce el efecto opuesto al receptor CB1: activa la liberación de neurotransmisores en las células presinápticas. También se encuentra en la periferia del tracto gastrointestinal, en los osteoblastos y adipocitos. ${ }^{6}$

\section{Ligandos}

Del cerebro del cerdo se ha aislado el ligando del receptor cannabinoide endógeno o endocannabinoide, el $\mathrm{N}$-araquidonoiletanolamina (AEA) o anandamida. ${ }^{11}$ Del tejido intestinal canino se ha aislado el ligando 2-araquidonoilglicerol (2-AG). ${ }^{9}$

Cuando una neurona presináptica libera un neurotransmisor para estimular a una neurona postsináptica, los ligandos se difunden pasivamente de forma retrógrada hacia la célula presináptica; se unen al receptor cannabinoide CB1 e inician la cascada que inhibe la liberación de neurotransmisores de la célula presináptica y de esta manera se detiene la transmisión sináptica ${ }^{10-12}$ Por lo tanto, AEA puede ser más activo en procesos agudos y 2-AG en crónicos.

\section{Enzimas biosintéticas y degradantes}

Son el tercer componente principal del sistema endocannabinoide, modulan la síntesis y la degradación de los ligandos endógenos, lo que influye en la funcionalidad del sistema: $\mathrm{N}$-araquidonoiletanolamina (AEA) o anandamida, diacilglicerol lipasa (DAGL) $\alpha 0$ $\beta$, amida hidrolasa de ácidos grasos (FAAH) de las enzimas serina hidrolasa y monoacilglicerol lipasa (MAGL). Todavía hay mucho que aprender sobre 
Tabla 1. Sistema endocannabinoide

\begin{tabular}{|c|c|c|c|c|}
\hline Cannabinoide & Receptor & Localización & Enfermedad & Efecto $^{8}$ \\
\hline SEC & CB1, TRPV1 & $\begin{array}{l}\text { Cerebro y } \\
\text { terminales nerviosas }\end{array}$ & Dolor y migraña & $\begin{array}{l}\text { Inhibe la liberación de glutamato, reduce el } \\
\text { calcio intracelular, promueve vasodilatación } \\
\text { y función sistólica conservada. Estimula la } \\
\text { producción de capsaina }\end{array}$ \\
\hline \multirow[t]{7}{*}{ Cannabidiol } & $\begin{array}{l}\text { CB1, CB2, } \\
\text { GPR55, FAAH }\end{array}$ & $\begin{array}{l}\text { Cerebro y } \\
\text { tallo cerebral }\end{array}$ & Epilepsia & $\begin{array}{l}\text { Receptor de } 5-\mathrm{HT} 1 \mathrm{~A} \text { y receptor de glicina } \alpha 3 \\
\text { y } \alpha 1 \text {, así como de calcio intracelular }\end{array}$ \\
\hline & CB1 & Cerebro & $\begin{array}{l}\text { Traumatismo craneoencefálico } \\
\text { grave y enfermedad vascular } \\
\text { cerebral }\end{array}$ & Inhibe la liberación de glutamato \\
\hline & CB1 & Tracto gastrointestinal & Trastornos digestivos & $\begin{array}{l}\text { Activa músculo liso y secreción de líquidos } \\
\text { digestivos }\end{array}$ \\
\hline & CB1 & & Alteración del sueño & Neuromodula \\
\hline & $\mathrm{CB} 1, \mathrm{CB} 2$ & Hígado y páncreas & Obesidad mórbida y diabetes & Resistencia a la insulina \\
\hline & $\mathrm{CB} 1, \mathrm{CB} 2$ & & Cáncer & $\begin{array}{l}\text { Retarda proceso de proliferación celular y } \\
\text { angiogénesis }\end{array}$ \\
\hline & $\begin{array}{l}\text { AEA disminuye y } \\
\text { 2-AG aumenta }\end{array}$ & $\begin{array}{l}\text { Eje } \\
\text { hipotalámico-pituitario }\end{array}$ & Respuesta inmediata al estrés & Plasticidad sináptica y memoria \\
\hline Cannabidiol & & $\begin{array}{l}\text { Disminuye la actividad } \\
\text { de la amígdala } \\
\text { izquierda }\end{array}$ & Ansiedad & \\
\hline \multirow[t]{4}{*}{ THC } & & $\begin{array}{l}\text { Alteración en áreas } \\
\text { límbicas y corticales }\end{array}$ & Psicosis & \\
\hline & $\mathrm{CB} 1, \mathrm{CB} 2$ & Inhibe la microglía & Enfermedad de Alzheimer & $\begin{array}{l}\text { Neuroprotección a través del factor } \\
\text { neurotrófico }\end{array}$ \\
\hline & $\begin{array}{l}\mathrm{CB} 1, \mathrm{CB} 2, \mathrm{AEA} \\
2 \mathrm{AG}\end{array}$ & Ganglios basales & Enfermedad de Parkinson & Modula la señalización de la dopamina \\
\hline & CB1, CB2, FAAH & Corteza/hipocampo & Enfermedad de Huntington & Disminución de la función del receptor ECS \\
\hline THC y CBD & CB1, CB2 & $\begin{array}{l}\text { Médula espinal y tallo } \\
\text { cerebral }\end{array}$ & Esclerosis múltiple & Espasticidad y dolor \\
\hline
\end{tabular}

$\mathrm{SEC}=$ sistema endocannabinoide, $\mathrm{THC}=$ delta-9-tetrahidrocanabinol, $\mathrm{CB} 1=$ receptor acoplado a proteína $\mathrm{G}(\mathrm{GPCR}), \mathrm{CBD}=$ cannabidiol, $\mathrm{CB} 2=$ receptor de cannabinoides tipo 2 , $2 A G=$ ligando 2-araquidonoilglicerol, TRPV1 = receptor transitorio potencial vanilloid-1, AEA = N-araquidonoiletanolamina, FAAH = amida hidrolasa de ácidos grasos.

estos procesos: FAAH y MAGL son prometedores para la manipulación farmacológica de las vías de señalización del sistema endocannabinoide.

\section{Relación de los fitocannabinoides y el sistema endocannabinioide}

Los cannabinoides exógenos o fitocannabinoides de origen natural ${ }^{7,8}$ interactúan con el sistema endocannabinoide. El compuesto cannabinoide por excelencia, $\triangle 9$-THC, se une a los receptores CB1 y CB2 como un agonista parcial débil; se cree que la unión de CB1 es principalmente responsable de los efectos intoxicantes y psicotrópicos de la planta del cannabis. El cannabidiol tiene poca afinidad por los receptores CB1 o $\mathrm{CB} 2$, inhibe la unión del tetrahidrocannabinol a CB1, puede potenciar o inhibir una variedad de efectos finales sobre las células, desde la modulación de los niveles de calcio intracelular hasta la capacidad de ejercer propiedades antioxidantes; se ha demostrado que su activación reduce la liberación de citocinas proinflamatorias, lo que sugiere su papel en la modulación de la inflamación y respuestas nociceptivas a infección y lesión. A diferencia del tetrahidrocannabinol, el cannabidiol no induce un efecto intoxicante o psicotrópico en el paciente o usuario. ${ }^{13,14}$

La activación del receptor TRPV1 modula una vía de estímulo del dolor diferente de las vías ocupadas por los endocannabinoides. TRPV1 también es de uso potencial en el tratamiento de las convulsiones y la epilepsia, sin embargo, la forma precisa en que los 
canales TRP influyen en la epilepsia sigue sin estar clara.

El cannabis es comúnmente consumido vía inhalación o ingestión y menos frecuentemente a través de preparaciones oftálmicas, rectales, sublinguales y dérmicas. ${ }^{15}$ Es posible que el uso persistente del cannabis resulte en déficits neurocognitivos duraderos y afecte la estructura y función del cerebro. ${ }^{16}$ Estas alteraciones neurofisiológicas deben considerarse tanto en investigación como en aplicaciones clínicas. ${ }^{17}$ Hay estudios que muestran una relación directa entre el riesgo de esquizofrenia y el consumo de cannabis. ${ }^{18}$

A pesar de lo anterior, no hay consenso en la literatura científica respecto a la naturaleza exacta, magnitud y duración de los cambios cerebrales, que pueden depender de la frecuencia, cantidad, duración y edad al inicio del uso del cannabis, así como la duración de la abstinencia. Además, tampoco está claro si las alteraciones identificadas son consecuencia de o preceden al consumo. Se necesitan estudios longitudinales adicionales que evalúen muestras más grandes, particularmente antes del inicio del consumo de cannabis, para determinar una ruta causal entre el consumo del cannabis y estas alteraciones. Algo parecido sucedió con el tabaco: fue necesario que pasaran 30 o más año de su consumo para conocer las consecuencias sobre la salud. ${ }^{19}$

El uso de cannabis medicinal tiene implicaciones legales, éticas y sociales. En Estados Unidos, la marihuana está actualmente reconocida por la Drug Enforcement Agency (DEA) como una sustancia controlada de tipo I. La Food and Drugs Administration (FDA) no aprueba la marihuana como una droga segura ni efectiva para ninguna indicación. La European Medicines Agency (EMA) le da designación de "huérfanos" al tetrahidrocannabinol y al cannabidiol, con la consecuente utilidad y seguridad terapéutica en enfermedades raras. ${ }^{20}$

La marihuana debe ser procesada en un laboratorio para extraer el tetrahidrocannabinol, responsable de los efectos intoxicantes y psicotrópicos del cannabis, y dejar el cannabidiol, el producto que se puede comercializar. Los productos del cannabis pueden contaminarse por la preparación y almacenamiento incorrectos y desarrollar cualquier microorganismo, particularmente bacterias y hongos (moho). Existen informes de contaminación bacteriana con Salmonella y Enterobacter, Streptococcus y Klebsiella, así como de casos de esporas de hongos, incluidas cepas de Aspergillus. ${ }^{21}$
Algunos estudios conceden al cannabidiol un gran potencial para el uso terapéutico como antiepiléptico, analgésico, ansiolítico, antipsicótico, antiinflamatorio y neuroprotector. ${ }^{22}$ Hay hallazgos inconsistentes de la eficacia de cannabinoides en el dolor neuropático y espasmos dolorosos en la esclerosis múltiple, así como respecto a la tolerabilidad y seguridad de los medicamentos con base en el cannabis para cualquier dolor crónico. ${ }^{23}$ También hay limitada evidencia de su asociación con el riesgo de efectos adversos a corto plazo y potenciales efectos adversos mentales, tales como la psicosis. ${ }^{24}$

No existen suficientes ensayos aleatorizados bien diseñados para confirmar los beneficios y daños del uso del cannabis. Los efectos adversos de los cannabinoides no son claros porque existe poca evidencia metodológica que los cuantifique. Mucho de lo que se sabe sobre los efectos adversos del cannabis medicinal viene de estudios relativos al uso recreativo de la marihuana. Por lo tanto, son necesarias investigaciones a largo plazo en torno a la evaluación de los efectos adversos por el uso crónico del cannabis medicinal, para evaluar concluyentemente los riesgos en un periodo extendido de uso. ${ }^{25}$

En vista de las considerables limitaciones de los estudios disponibles y de acuerdo con las conclusiones de varios metaanálisis, no es posible recomendar el cannabis ni la medicina basada en cannabis como tratamiento del dolor musculoesquelético, artritis o fibromialgia. Hay una necesidad imperiosa de ensayos clínicos bien controlados de mayor amplitud. ${ }^{22,26}$

\section{Conclusión}

El uso medicinal del cannabis necesita más evidencias obtenidas con criterio científico, debido a que no se han efectuado estudios clínicos bien diseñados con muestras sustanciales y tiempo de duración suficiente, factores imprescindibles para la calidad y fiabilidad de las investigaciones.

\section{Bibliografía}

1. Kuddus M, Ginawi IAM, Al-Hazimi A. Cannabis sativa: an ancient wild edible plant of India. Emir J Food Agric. 2013;25:736-745.

2. Elsohly MA, Slade D. Chemical constituents of marijuana: The complex mixture of natural cannabinoids. Life Sci. 2005;78:539-548.

3. Schultes RE, Klein WM, Plowman T, Lockwood TE. Cannabis: an example of taxonomic neglect. Bot Mus Lealf Harv Univ.1974;23:337-367.

4. Williamson EM, Evans FJ. Cannabinoids in clinical practice. Drugs. 2000;60:1303-1314

5. Small E. Evolution and classification of cannabis sativa (marijuana, hemp) in relation to human utilization. Bot Rev. 2015;81:189-294. 
6. Lannotti FA, Di Marzo V, Petrosino S. Endocannabinoids and endocannabinoid-related mediators: targets, metabolism and role in neurological disorders. Prog Lipid Res. 2016;62:107-128.

7. Russo E, Guy GW. A tale of two cannabinoids: the therapeutic rationale for combining tetrahydrocannabinol and cannabidiol. Med Hypotheses. 2006;66:234-246

8. Russo EB. Cannabidiol claims and misconceptions. Trends Pharmaco Sci. 2017;38:198-201

9. Matsuda LA, Lolait SJ, Brownstein MJ, Young AC, Bonner TI. Structure of a cannabinoid receptor and functional expression of the cloned cDNA. Nature. 1990;346:561-564

10. Di Marzo V, Piscitelli F. The endocannabinoid system and its modulation by phytocannabinoids. Neurotherapeutics. 2015;12:692-698.

11. Devane WA, Hanus L, Breuer A, Pertwee RG, Stevenson LA, Griffin G, et al. Isolation and structure of a brain constituent that binds to the cannabinoid receptor. Science. 1992;258:1946-1949.

12. Morena M, Patel S, Bains JS, Hill MN. Neurobiological interactions between stress and the endocannabinoid system. Neuropsychopharmacology. 2016;41:80-102.

13. Russo EB, Burnett A, Hall B, Parker KK. Agonistic properties of cannabidiol at 5-HT1a receptors. Neurochem Res. 2005;30:1037-1043.

14. Benito C, Tolon RM, Pazos MR, Núñez E, Castillo Al, Romero J. Cannabinoid $\mathrm{CB} 2$ receptors in human brain inflammation. Br $\mathrm{J}$ Pharmacol. 2008;153:277-285.

15. Dryburgh LM, Bolan NS, Grof ChPL, Galettis P, Schneider J, et al. Cannabis contaminants: sources, distribution, human toxicity and pharmacologic effects. Br J Clin Pharmacol. 2018;84:2468-2476.

16. Iversen L. Cannabis and the brain. Brain. 2003;126:1252-1270.
17. Filbey FM, Aslan S, Lu H, Peng SL. Residual effects of THC via novel measures of brain perfusion and metabolism in a large group of chronic cannabis users. Neuropsychopharmacology. 2018;43:700-707.

18. Pasman JA, Verweij KJH, Gerring Z, Stringer S, Sanchez-Roige S, Treur JL, et al. GWAS of lifetime cannabis use reveals new risk loci, genetic overlap with psychiatric traits, and a causal influence of schizophrenia. Nat Neurosci. 2018;21:1161-1170.

19. Nader DA, Sánchez ZM. Effects of regular cannabis use on neurocognition, brain structure, and function: a systematic review of findings in adults. Am J Drug Alcohol Abuse. 2018;44:4-18.

20. Codas M, Haurón G, Achucarro D, Usher F, Pescador-Ruschel MA, Sebriano M, et al. Cannabis en el dolor crónico. Rev Virtual Soc Parag Med Int. 2018:5:12-18.

21. Dryburgh LM, Bolan NS, Grof CPL, Galettis P, Schneider J, Lucas CJ, et al. Cannabis contaminants: sources, distribution, human toxicity and pharmacologic effects. Br J Clin Pharmacol. 2018;84:2468-2476.

22. Bridgeman MB, Abazia DT. Medicinal cannabis: history, pharmacology, and implications for the acute care setting. PT. 2017;42:180-188.

23. Häuser W, Petzke F, Fitzcharles MA. Efficacy, tolerability and safety of cannabis-based medicines for chronic pain management-an overview of systematic reviews. Eur J Pain. 2018;22:455-470.

24. Nugent SM, Morasco BJ, O’Neil ME, Freeman M, Low A, Kondo K, et al. The effects of cannabis among adults with chronic pain and an overview of general harms: a systematic review. Ann Intern Med. 2017;167:319-331.

25. Karila L, Roux P, Rolland B, Benyamina A, Reynaud M, Aubin HJ, et al. Acute and long-term effects of cannabis use: a review. Curr Pharm Des. 2014:20:4112-4118.

26. Perrot S, Trouvin AP. Cannabis for musculoskeletal pain and arthritis: evidence is needed. Joint Bone Spine. 2018;86:1-3. 